\title{
3D Atom Probe: Chemical Analysis With (Near) Atomic Resolution
}

\author{
F. Danoix, F. Vurpillot, W. Lefebvre, D. Blavette
}

Groupe de Physique des Matériaux, UMR 6634 CNRS-Université de Rouen

Avenue de l’Université, 76801 Saint Etienne du Rouvray Cedex, France

Since it's invention in the early 50's, field ion microscopy (FIM) allowed a true atomic resolution inspection of metallic materials. Lattice related features could be imaged for the very first time. Unfortunately, no chemical information can be obtained with the field ion microscope. This limitation was overcame in the late 60's by the addition to the field ion microscope of a time of flight mass spectrometer, equipped with a single atom detection system, giving birth to the atom probe (AP) technique [1]. Combining AP and FIM, the determination of the chemical nature of selected individual atoms became possible. Even if very exciting, the atom by atom process was very tedious, and routine experiments almost impossible to realise. The use of position sensitive detection system in the late 80' [2] opened a new way to approach crystal lattice reconstruction with chemical information. The analysis of a tungsten specimen in the $<111>$ direction was realised. Using a Fourrier transform over the analysed volume, spots related to low index crystallographic directions were imaged, and reverse Fourrier transform technique was used to give an image of the BCC specimen lattice structure. With a confidence level higher than $90 \%$, true atomic positioning and chemical identification of each individual atom is reached.

Even if lattice reconstruction has not been achieved for most of the metallic alloys, the spatial resolution of 3D AP is of the highest, well within the subnanometric range. Major scientific results have been obtained recently with this technique [3]. An example of the capabilities of the technique in materials science is the analysis of the nanostructured soft magnetic material FINEMET. The 3D reconstructions of the analysed final nanostructure, as a result of solute atom distribution during crystallisation, gives access to a better understanding of the magnetic properties of this material.

One of the key issues of this AP analysis remains specimen preparation. In particular, when the feature of interest is spatially limited, standard electropolishing preparation methods fail. The emergence of the focused ion beam (FIB) technique opens new areas of investigation for the atom probe, as recently demonstrated in the case of multilayers [4]. Some examples of specimen preparation using FIB will be shown.

\section{References}

[1] M.K. Miller, A. Cerezo, M.G. Hetherington, and G.D.W. Smith, Atom Probe Field Ion Microscopy, Clarendon Press, Oxford (1996)

[2] M.K. Miller, Atom Probe Tomography: Analysis at the atomic scale. Kluwer Academic/Plenum Press, Dordrecht/New York (2000)

[3] D. Blavette, E. Cadel, A. Fraczkiewicz, A. Menand, Science Dec. 17 (1999) 2317

[4] D.J Larson, P.H.Clifton, N.Tabat, A.Cerezo, A.K. Petford-Long, R.L.Martens and T.F.Kelly, Appl. Phys. Let., 77 (2000) 726

e-mail: frederic.danoix@univ-rouen.fr 

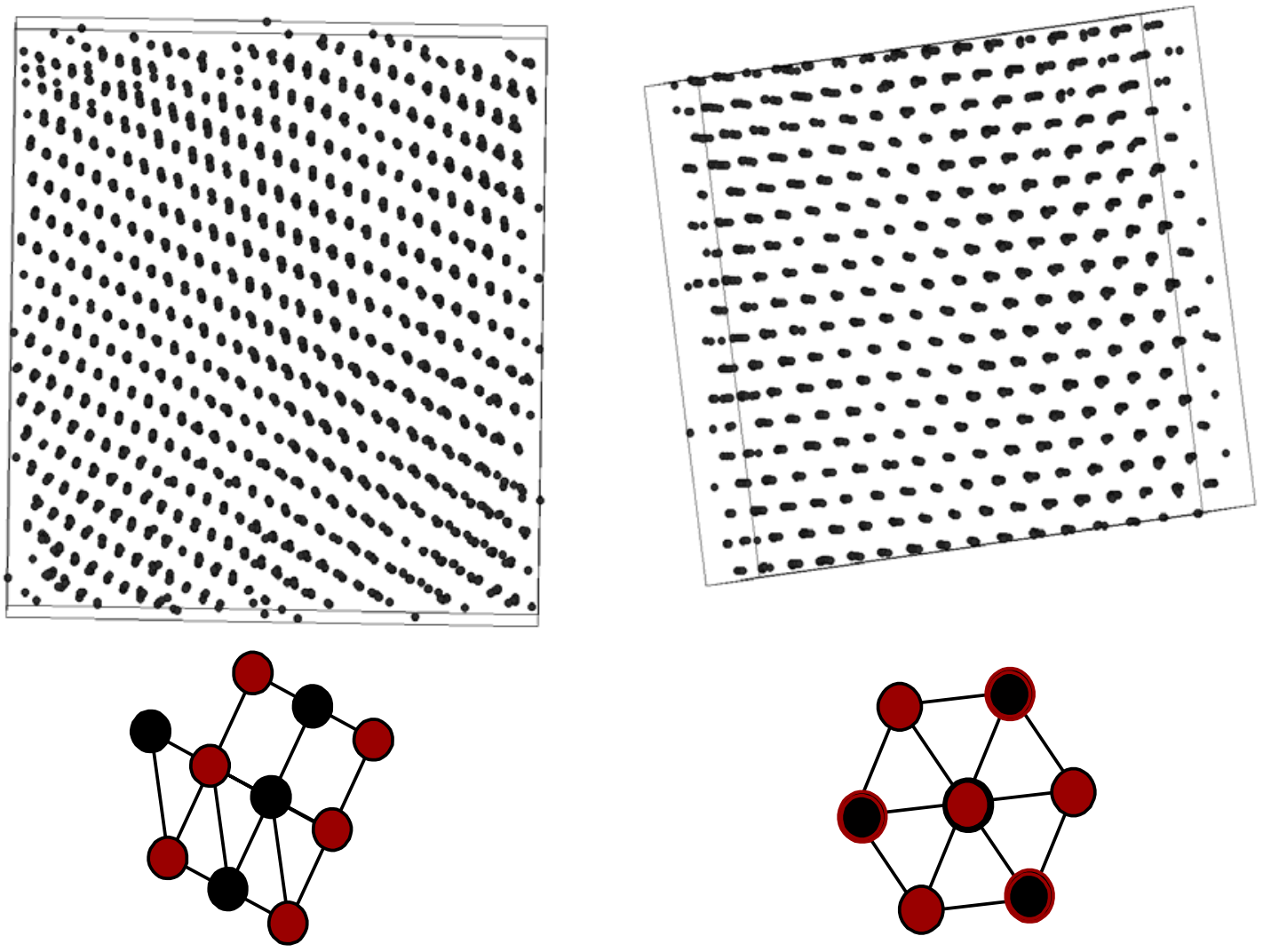

Fig. 1. lattice reconstruction in a tungsten specimen (each dot is an individual atom) a) front view along (011) zone axis b) side view along (111) zone axis

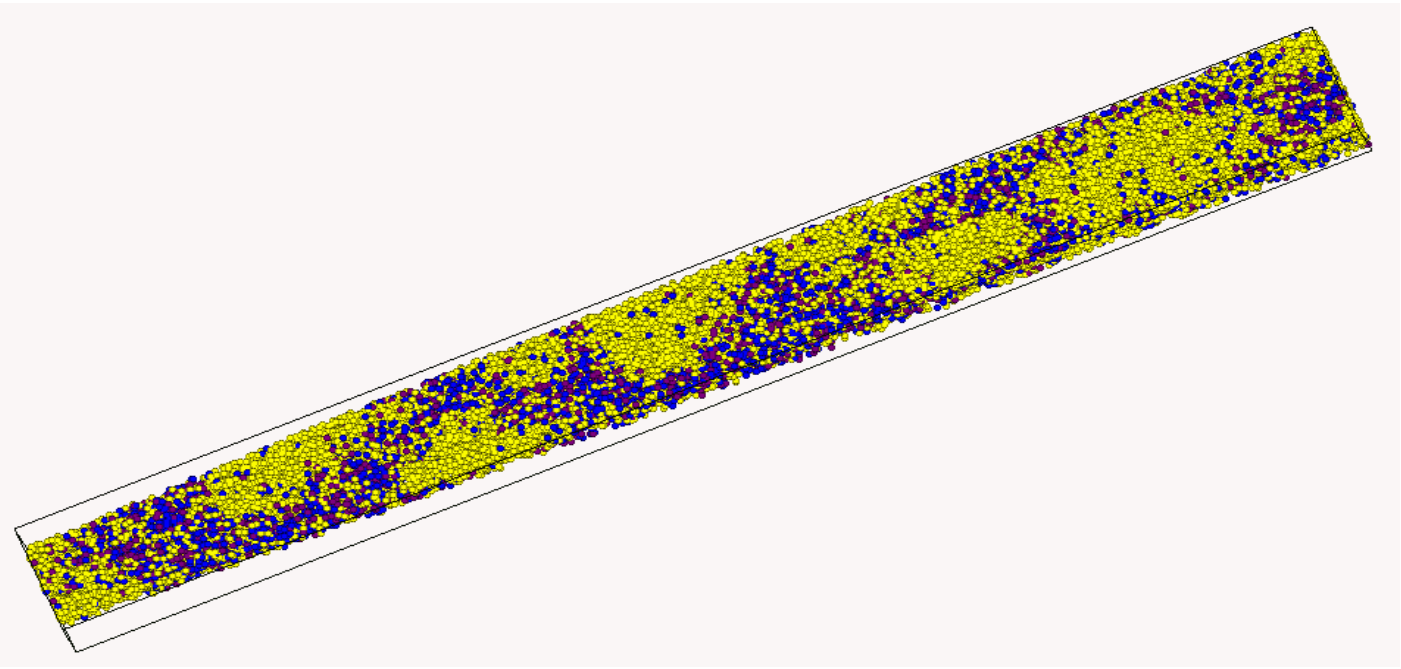

Fig. 2. 3D reconstruction of a nanocrystalline FINEMET alloy. The analysed volume is $14 \times 14 \times 130 \mathrm{~nm}^{3}$. Yellow dots are Si atoms, blue dots are B and purple ones are $\mathrm{Nb}$. 